\title{
Time to study time: informing the future of anaphylaxis observation duration
}

\author{
Roland Bingisser ${ }^{1,2} \cdot$ Brett Burstein $^{3,4}$
}

(c) The Author(s), under exclusive licence to Canadian Association of Emergency Physicians (CAEP)/ Association Canadienne de Médecine d'Urgence (ACMU) 2021

Keywords Anaphylaxis · Allergic reaction · Observation time

Anaphylaxis is a potentially life-threatening systemic allergic reaction. Biphasic reactions [1], i.e., return of symptoms following a period of full clinical resolution, are well documented, but vary widely in terms of definitions [2], severity, and reported frequency. Guidelines regarding observation duration for anaphylaxis and risk factors predicting biphasic reactions are similarly variable [3]. An improved ability to identify patients most likely to benefit from prolonged observation would optimize care and resource utilization, but must be based on more robust evidence than is currently available.

In this issue of CJEM, Simard and colleagues present a systematic review summarizing the association between biphasic anaphylaxis and potential risk factors [4]. The review identified 21 adult and pediatric studies and found five recurrent predictors (time to first epinephrine, history of anaphylaxis, symptom severity, number of epinephrine doses, and unknown trigger), and five additional predictors less consistently reported and less well defined (time to symptom resolution, arrival by ambulance, administration of $\beta$-agonists, young age and age $6-9$ years among pediatric patients).

\section{Brett Burstein}

brett.burstein@mail.mcgill.ca

1 Emergency Medicine, Department of Acute Medicine, University Hospital, Basel, Switzerland

2 Department of Clinical Research, Emergency Medicine Research, University of Basel, Basel, Switzerland

3 Division of Pediatric Emergency Medicine, Department of Pediatrics, Montreal Children's Hospital, McGill University Health Centre, 1001 Decarie Blvd, Montreal, QC H4C 3J1, Canada

4 Department of Epidemiology, Biostatistics and Occupational Health, McGill University, Montreal, QC, Canada
A strength of this study is the inclusion of a large number of studies and a comprehensive analysis of potential risk predictors. Importantly, however, just 3 of 21 included studies were prospective, with an additional 4 using both retrospective and prospective designs. Also complicating interpretations of the findings, 10 of 21 studies contained identical or overlapping patients. While the authors took caution to assess bias, studies with a clear risk of bias were not excluded. They also very appropriately separated studies that used different case definitions for biphasic reactions, and analyzed risk predictors restricted to studies defining "clinically significant" biphasic reactions. However, three of six studies focusing on significant reactions were at high risk of bias and the remaining three were at an unclear risk of bias.

Perhaps the most significant limitation to incorporating the identified risk factors directly into clinical practice is that this review was not designed to adjust for confounding. It would be ideal to be able to control for patient- and study setting-level covariates, several of which are also likely to be colinear. The ranked list of risk factors were those most frequently reported among patients with biphasic reactions. However, the study design did not permit multivariable modeling to assess the relative importance of each factor, particularly for the outcome most of interest, clinically significant biphasic reactions. The authors conclude that the identified predictors "should not be treated as prolonged observation criteria but rather as the first steps toward creating an early discharge tool following anaphylaxis."

This analysis leads to two different interpretations and consequences for future studies:

Interpretation 1: Pragmatic approach: It may be reasonable to next conduct an interventional trial informed by these study results using the identified risk factors, for example in a multicenter trial in which patients are discharged with none 
of the five main risk factors. Arguments for this approach are the relatively low risk of biphasic reactions in general [1] and a recent recommendation to self-administer epinephrine at home and only seek ED care if symptoms do not resolve [5]. This approach could be cost-effective, in terms of both study design and patient-care, unifying the different recommendations into a fast and frugal clinical strategy with standardized medication plans for all significant anaphylactic reactions. Disadvantages are safety concerns and persisting uncertainty about the necessity of certain medications, such as steroids [6] and antihistamines [7].

Interpretation 2: Academic approach: An alternative strategy might instead focus on robustly deriving and validating the role of potential predictors using a prospective design. This approach could use the analysis by Simard as a starting point, while collecting data on a larger number of potential predictor variables. The primary arguments for the academic approach are superior information on a large number of potential risk factors and the ability to discern differences between adult and pediatric populations. This is a common approach similar to studies designed for the prediction of infrequent outcomes [8,9], and would offer a more robust foundation for subsequent interventions.

Several important questions remain unanswered.

First, what is the value and cost of reducing observation times? Second, should we focus on observation only in high risk situations, allocating observation resources to the estimated $10 \%$ high-risk patients, instead of trying to determine the optimal observation windows? Third, what is the acceptable complication or readmission rate after early discharge?

Though it may be debated whether the data gathered are solid enough to start intervention trials, some guidance may be found among the recent recommendations of the Joint Task Force for Practice Parameters of the American Academy of Allergy, Asthma \& Immunology and the American College of Allergy, Asthma and Immunology [10]. These recommend that clinicians incorporate severity of anaphylaxis and/or the administration of $>1$ dose of epinephrine as a guide to determine the risk for biphasic reactions (Conditional recommendation; very low certainty rating of the evidence). Prompt and adequate treatment of anaphylaxis appears central to reducing biphasic anaphylaxis risk [10]. While the possibility of biphasic anaphylaxis may be lower among patients without these features, it is important to educate all patients regarding the chance of a biphasic reaction, as well as avoiding known triggers, identifying early symptoms, using auto-injector epinephrine, and timely follow-up with an allergist.

Is it time to further study observation time? Ideally, in clinical practice the need for prolonged observation should be individualized using evidence-based risk-stratification. Future studies attempting to resolve this question must either apply our current understanding of risk factors for biphasic reactions to inform interventional trials, or attempt to more robustly define risk factors and their relative importance.

\section{Declaration}

Conflict of interest The authors have no conflicts of interest to disclose.

\section{References}

1. Kraft M, Scherer Hofmeier K, Rueff F, et al. Risk factors and characteristics of biphasic anaphylaxis. J Allergy Clin Immunol Pract. 2020;8(10):3388-95 e6.

2. Dribin TE, Sampson HA, Camargo CA Jr, et al. Persistent, refractory, and biphasic anaphylaxis: a multidisciplinary Delphi study. J Allergy Clin Immunol. 2020;146(5):1089-96.

3. Burroughs-Ray DC, Wells DR, Dennis MM, et al. Clinical guideline highlights for the hospitalist: anaphylaxis management in adults and children. J Hosp Med. 2021;16(2):98-9.

4. Simard. Factors influencing emergency department observation time following anaphylaxis: a systematic review. Can J Emerg Med. 2021.

5. Casale TB, Wang J, Nowak-Wegrzyn A. Acute at home management of anaphylaxis during the Covid-19 pandemic. J Allergy Clin Immunol Pract. 2020;8(6):1795-7.

6. Alqurashi W, Ellis AK. Do corticosteroids prevent biphasic anaphylaxis? J Allergy Clin Immunol Pract. 2017;5(5):1194-205.

7. Sheikh A, ten Broek V, Brown SG, et al. H1-antihistamines for the treatment of anaphylaxis with and without shock. Cochrane Database Syst Rev. 2007;1:CD006160.

8. Osmond MH, Klassen TP, Wells GA, et al. CATCH: a clinical decision rule for the use of computed tomography in children with minor head injury. CMAJ. 2010;182(4):341-8.

9. Stiell IG, Wells GA, Vandemheen K, et al. The Canadian CT Head Rule for patients with minor head injury. Lancet. 2001;357(9266):1391-6.

10. Shaker MS, Wallace DV, Golden DBK, et al. Anaphylaxisa 2020 practice parameter update, systematic review, and Grading of Recommendations, Assessment, Development and Evaluation (GRADE) analysis. J Allergy Clin Immunol. 2020;145(4):1082-123. 